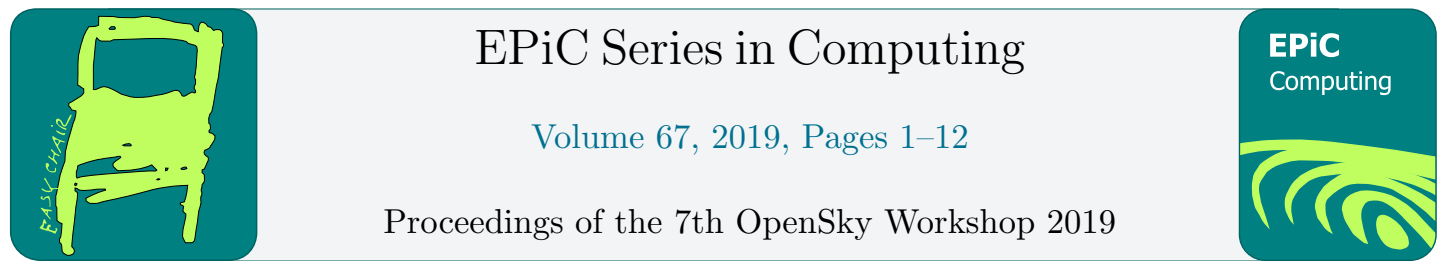

\title{
DFS ADS-B Implementation in High Density Radar Controlled Airspace - Experiences and Challenges
}

\author{
Stefan Stanzel \\ DFS Deutsche Flugsicherung GmbH, Langen, GERMANY \\ stefan.stanzel@dfs.de
}

\begin{abstract}
The German Air Navigation Service Provider (ANSP) DFS Deutsche Flugsicherung (DFS) is operating a surveillance infrastructure mainly based on radars and multilateration systems, which provides surveillance information and receives aircraft derived data. The implementation and use of the new surveillance technology Automatic Dependent Surveillance - Broadcast (ADS-B) has to provide benefits in terms of safety, performance or costs. DFS operations require a permanent double surveillance coverage for the area of responsibility. This requirement actually means triple surveillance coverage (in order to provide a seamless service also in case of system outages or planned down times). DFS is implementing ADS-B as the third surveillance layer to reduce costs. Each surveillance layer has to fulfil the performance and safety requirements independently. The fulfilment of safety requirements, integrity and continuity, will require validation of the passively received ADS-B information.

Data integrity and continuity need to be considered w.r.t. safety requirements, but may also have to address security. The integrity of ADS-B data can be ensured either by comparison with data from other surveillance sensors (dependent validation) or by analyzing the properties of the data itself (independent validation). The independent validation of the ADS-B data is a necessary prerequisite for providing a self-contained independent surveillance layer using the ADS-B technology. In a first step, DFS will implement ADS-B as a dependent layer: (1) for validation of the assumption on surveillance performance, (2) to gain experience on data integrity and data continuity, (3) to prepare possible mitigations e.g. against spoofing or jamming and (4) to recognize potential shortcomings in the airborne installations and identify possible mitigations. This first step will support necessary developments (e.g. ADS-B Validation Unit) to move with ADS-B towards the use as an independent surveillance layer reducing infrastructure costs and improve radio spectrum protection.
\end{abstract}




\section{Legislative Context}

The Surveillance Performance and Interoperability Requirements Implementing Rule SPI-IR 1207 was published in 2011 (SPI-IR 1207/2011 (EUROPEAN COMMISSION, 2011)) and amended in 2014 and 2017 is laying down requirements for the performance and the interoperability of air traffic surveillance for the single European sky. An ANSP ensures seamless operations within the airspace under its responsibility and at the boundary with adjacent airspaces by applying appropriate minimum requirements for the separation of aircraft. The performance requirements describes the accuracy, availability, integrity, continuity and timeliness of the surveillance data. The evaluation of the performance requirements is part of the surveillance system acceptance. The rules defined in the SPIIR 1207/2011 (EUROPEAN COMMISSION, 2011) will serve as the baseline for an operational implementation of ADS-B application in a radar environment (ADS-B RAD (EUROCAE, ED-161, Enhanced Air Traffic Services in Radar-Controlled Areas using ADS-B Surveillance (ADS-B RAD), 2009)). Air to Ground interoperability will be insured by "SPI-IR compliant" aircraft to be able to fly anywhere and receive a surveillance service. Therefore, ANSPs assume that any "SPI-IR compliant" aircraft provides data suitable for a surveillance service using any foreseen technology (Radar, WAM, ADS-B). ANSPs may implement any technology that meets the performance requirements, in other words there is no mandated specific surveillance technology. The Ground to Ground interoperability requires standardized data format when data is exchanged between systems. The current ADS-B equipage rate is measured continuously measured (e.g.: Frankfurt, Germany see Figure 1)

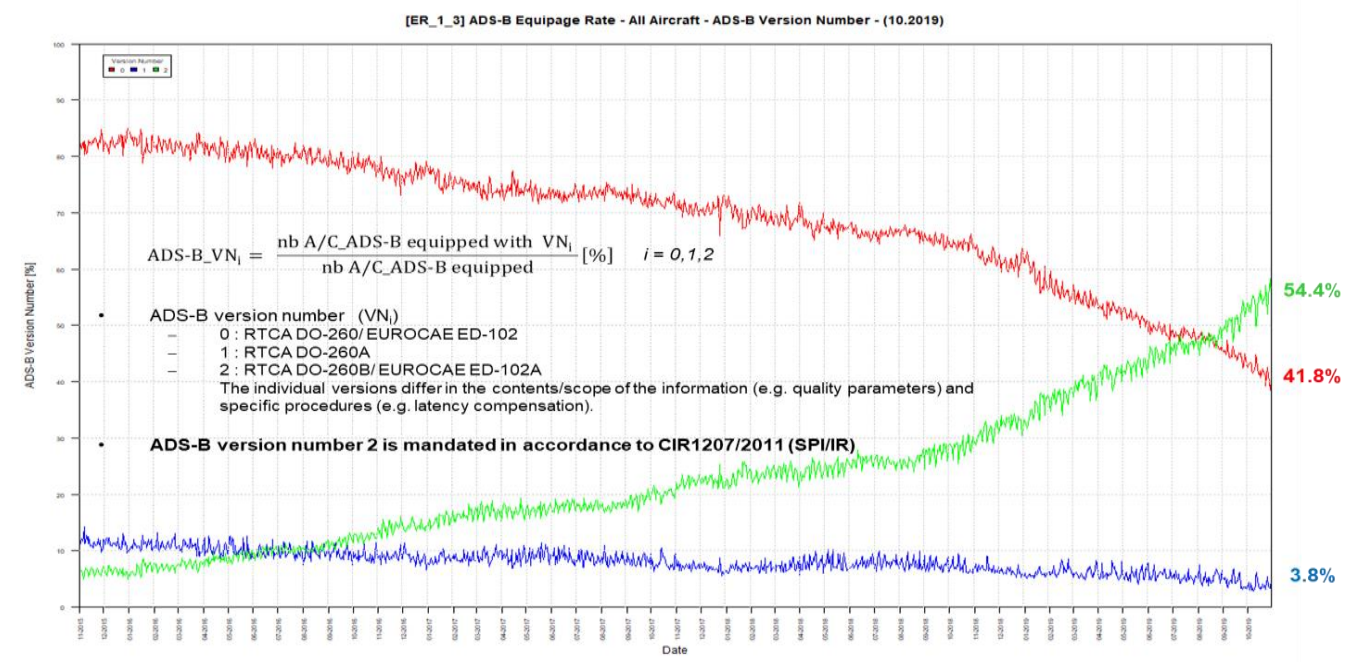

Figure 1 ADS-B Equipage Rate - Version Number

\section{Surveillance System - Logical Architecture}

The logical architecture of the surveillance system (see Figure 2) specifies the major functions of the spatial, aircraft and ground domains and their interaction, which is the basis for providing air traffic control services (ATS). 
For the spatial domain, it is assumed that the Global Navigation Satellite System (GNSS) is used. In this context, GNSS is a generic term for the use of existing and future global satellite systems, such as the US Global Positioning System (GPS), the GLObal NAvigation Satellite System (GLONASS) of the Russian Federation, Galileo of the European Union and the Chinese Compass.

The minimum surveillance equipment on board of aircraft comprises data sources (ICAO, 2012) (e.g. for the aircraft identification, 24-bit Mode $\mathrm{S}$ address, emergency indicator, flight level and position, as required; the full set of required data is described in the SPI/IR 1207/2011 (EUROPEAN COMMISSION, 2011)) and the associated processing and transmission functions by the transponder.

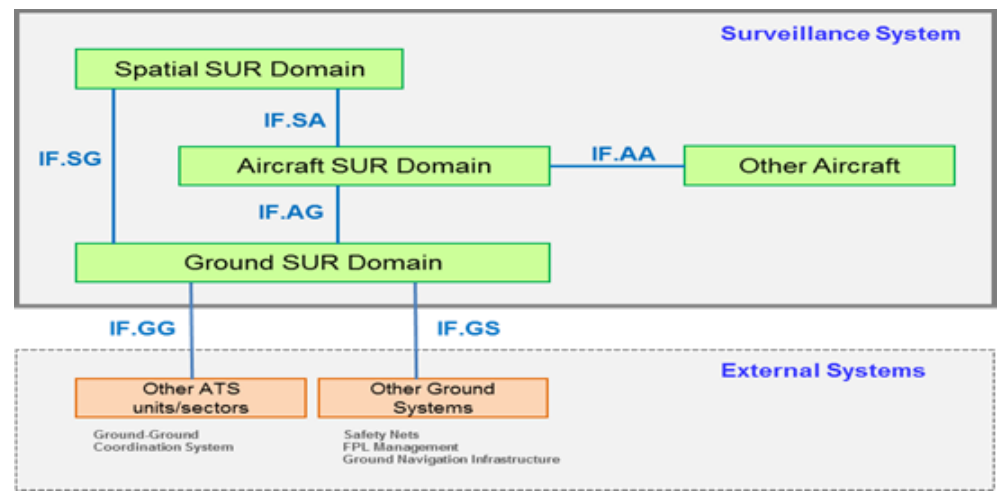

Figure 2 Logical architecture of a surveillance system

The minimum functional architecture of the ground surveillance domain comprises the following functions: surveillance sensor, surveillance data distribution, flight data processing and a display system with is the interface to the air traffic controller (ATCO) (see Figure 3).

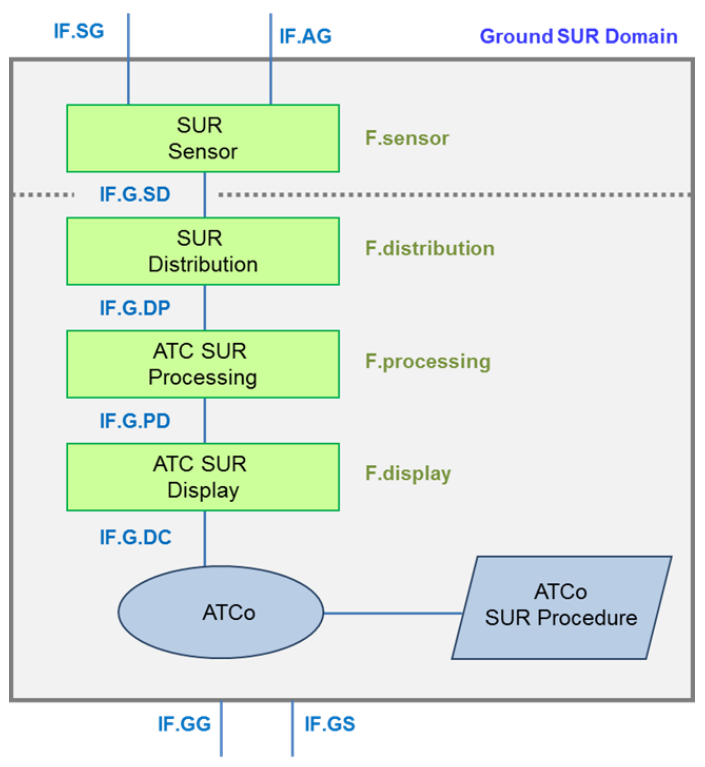

Figure 3 Ground surveillance domain 
When assessing the safety/security and performance of the ground domain, all interfaces and functions must be considered that are relevant from the transmission of data from the aircraft up to the point where they are displayed at the controller working position. Important aspects are, in particular, the time between sending and receiving as well as the environmental conditions.

\section{ADS-B in RADAR Controlled Airspace}

\subsection{Objectives}

The introduction of the surveillance technology ADS-B in the surveillance system may enhance Air Traffic Management (ATM) services by improving the overall quality of surveillance (i.e., ADS-B added to a single independent surveillance layer) such that an operational benefit may include a reduction in the applied separation standards from that applied in today's airspace. In some areas where, for example, single independent surveillance coverage is provided (ADS-B in non-Radar Airspace, ADS-B NRA), the lack of redundancy induces limited capacity due to the limited surveillance capabilities (tactical separation) and safety shortcomings. Lack of redundancy may be due to terrain, cost or physical limitations. Another example - which is more relevant for ANSPs in central Europe is where multi-radar presently exists but a radar is to be de-commissioned in the future and the radar replacement costs are not justified (ADS-B in Radar Airspace, ADS-B RAD).

\subsection{Implementation Approach}

A common approach from an existing cooperative independent surveillance infrastructure (Mode $\mathrm{A} / \mathrm{C} / \mathrm{S}$ radar) towards a cooperative dependent surveillance infrastructure (ADS-B) foresees the introduction of multilateration systems as a significant intermediate step. In addition to their primary purpose to provide surveillance information based on multilateration techniques, the Wide Area Multilateration (WAM) systems provide the capability of receiving ADS-B data as well. The ADS-B data are received from the remote units of the WAM sensor system and relayed to the central target processor. The target processor filters the ADS-B data in order to suppress duplicates, outdated information or geographical areas and provides a single ADS-B data stream to the surveillance data processing system.

Compared to single ADS-B Sensor a WAM system consists of a redundant array of ADS-B receiving systems, which is designed to improve the reliability of the ADS-B system and the robustness against disturbances. The WAM build-in verification function of ADS-B against multilateration data shall ensure that only valid ADS-B data are provided to the surveillance data processing systems. Additional functions provided by a WAM sensor system are the measurement of ADS-B probability of detection and coverage range as well as the monitoring of quality indicators.

\subsection{DFS ADS-B Implementation Steps}

In order to provide ADS-B surveillance data for a certain air traffic control application (e.g. center, tower) the complete corresponding ground surveillance chain is required to process the ADS-B data. Therefore, (1) the ADS-B coverage needs to be provided by deployment of ADS-B ground stations/systems (EUROCAE, ED-129B, Technical Specification for $1090 \mathrm{MHz}$ Extended Squitter ADS-B Ground System, 2009), (2) the distribution of the ADS-B data is ensured (DFS: SDDS-NG, ARTE), (3) the surveillance data processing systems (SDPS) are capable of processing ADS-B data (DFS: ARTAS, PHOENIX) and (4) the ADS-B data are appropriately presented to the ATCO (DFS: 
ICAS CWP). Usually the needs/capabilities of the Surveillance Data Processing System (SDPS) drive the requirements of the ground surveillance systems. Currently none of the operational DFS SDPS systems in use is capable of processing ADS-B data. However, the new DFS SDPS system (ICAS) will provide ADS-B functionalities. The rollout planning foresees that ICAS will be provided first for the upper airspace and later on for the lower airspace.

In order to establish the corresponding ADS-B coverage for the SDPS systems a stepwise approach for the deployment of ADS-B ground systems is planned: (1a) using the ground stations the PAM-FRA WAM system to provide initial ADS-B coverage for the upper airspace Karlsruhe, (1b) installation of additional ADS-B ground stations to cover the remaining upper airspace Karlsruhe, (2) additional ADS$B$ infrastructure installation to cover the lower airspace in the south of Germany and (3) expansion of the ADS-B infrastructure to cover the remaining lower airspace (see Figure 4).

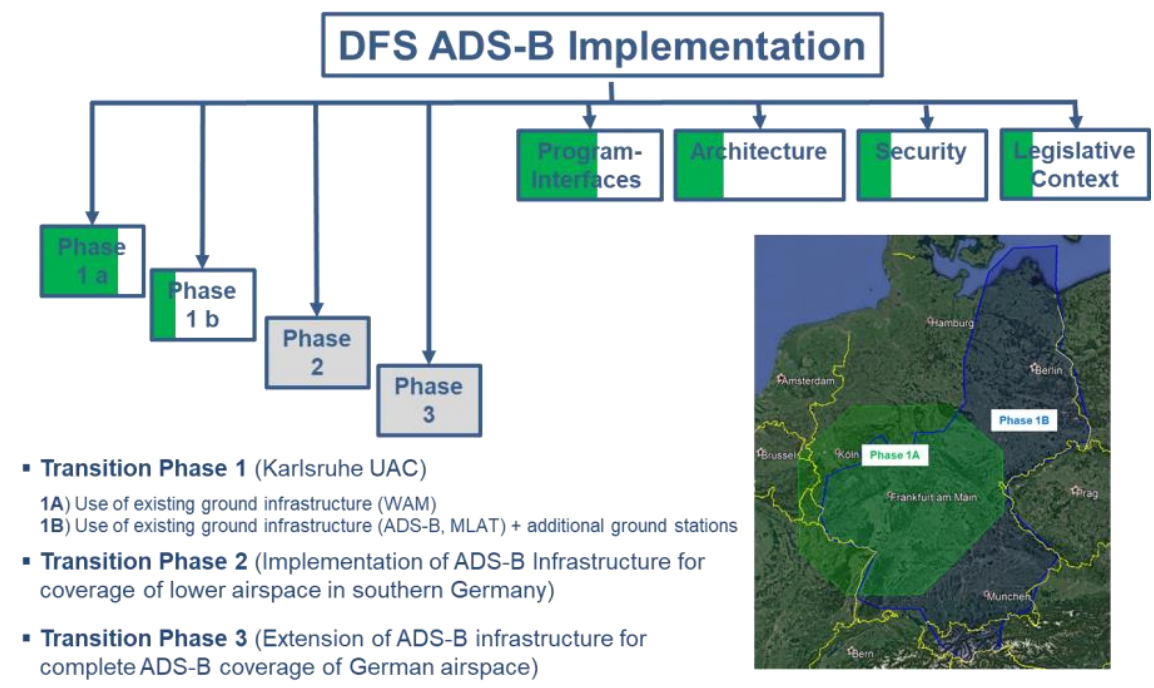

Figure 4 DFS ADS-B Implementation Steps

The operational use of ADS-B data in the DFS surveillance application requires that (1) only ADS B MOPS V2 (EUROCAE, ED-102A, Minimum Operational Performance Standards for $1090 \mathrm{MHz}$ Automatic Dependent Surveillance (Equivalent to RTCA DO-260B) (Equivalent to RTCA DO-260B: Minimum Operational Performance Standards for $1090 \mathrm{MHz}$ Automatic Dependent Surveillance), 2009) data are used (according to CIR (EU) 1207/2011, (2) target tracks are not established by ADS B only (until independent validation is implemented) and (3) a validation function needs to be established. The ADS-B data used for operational surveillance are filtered according quality parameters (see Figure 5).

The DFS design of the ADS-B infrastructure foresees that advanced, high performance Ground Stations will be used. These ADS-B Ground Stations will consist of multichannel receivers with sectorized antennas. In order to achieve an optimized coverage fulfilling high performance requirements (short Update Period (UP) using ADS-B data driven mode and high Probability of Update (PoU) even in environments with demanding radio field load). The goal is reduce life tine costs, which are mainly depending on site-specific costs (communication costs, rental charges, maintenance costs, etc.). 
Therefore, the reduction of the number of necessary sites in order to provide a ADS-B operational coverage is key for the economical introduction of this surveillance technology.
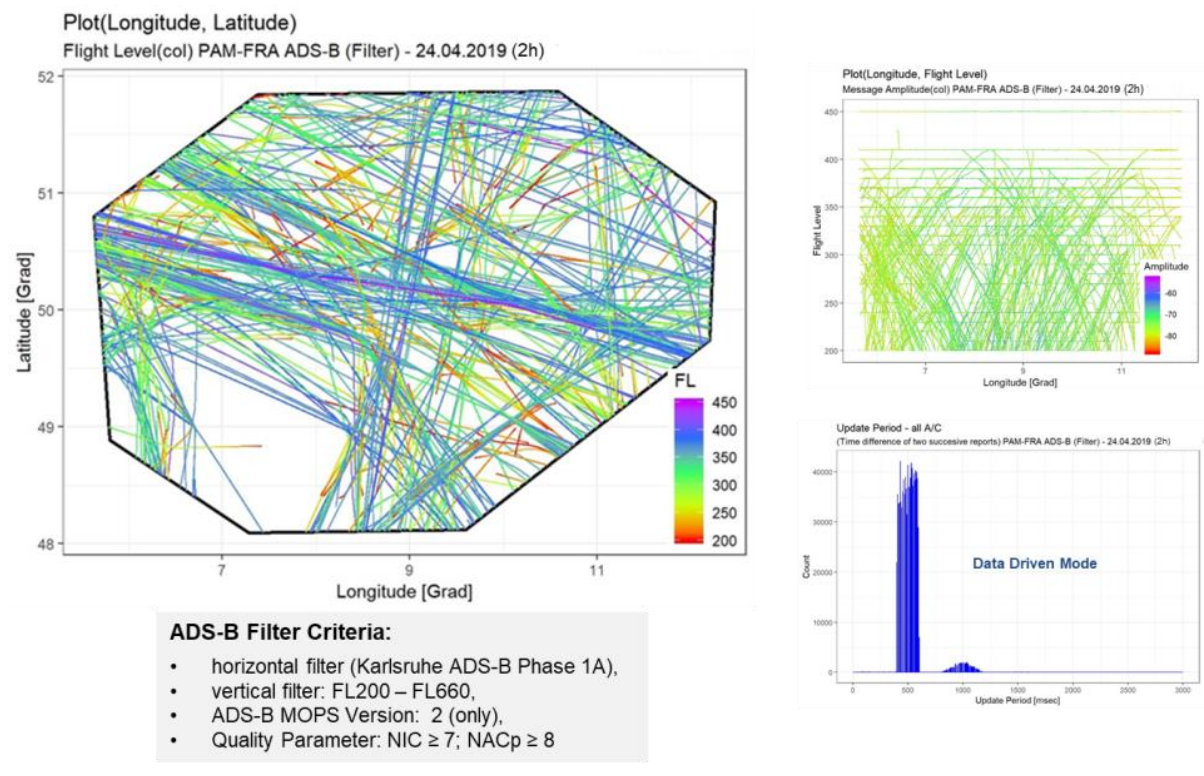

Figure 5 DFS ADS-B Implementation - Phase 1A

\section{ADS-B Performance Assessment (APA)}

The following performance metrics analyzed by DFS at surveillance sensor and surveillance data processing system (tracker) level using international evaluation standards (EUROCONTROL, 2012) (EASA, 2013) (Stanzel, 2009) are (see Figure 6):

- Probability of Update (at a defined Update Period)

The PoU is the rate of the number of received position updates and the number of expected position updates. The PoU is determined per target and addition per defined volume of airspace and as an overall value.

\section{- Horizontal Position Root-Mean-Square Error}

The error on the provided horizontal position is the 2D Euclidian distance between the horizontal position provided by the surveillance system under test and the reference horizontal position of the corresponding aircraft at the time when the updated position was output/delivered. This error takes into account any uncompensated latency between the time of applicability of the provided horizontal position and the time when the horizontal position was delivered to another system.

- Ground Speed Root-Mean-Square Error

The calculation of the reference aircraft velocity and angle will follow the same principle as for horizontal position error, i.e. the provided value will be compared with the reference value at the time the target report including track velocity data item was output. 

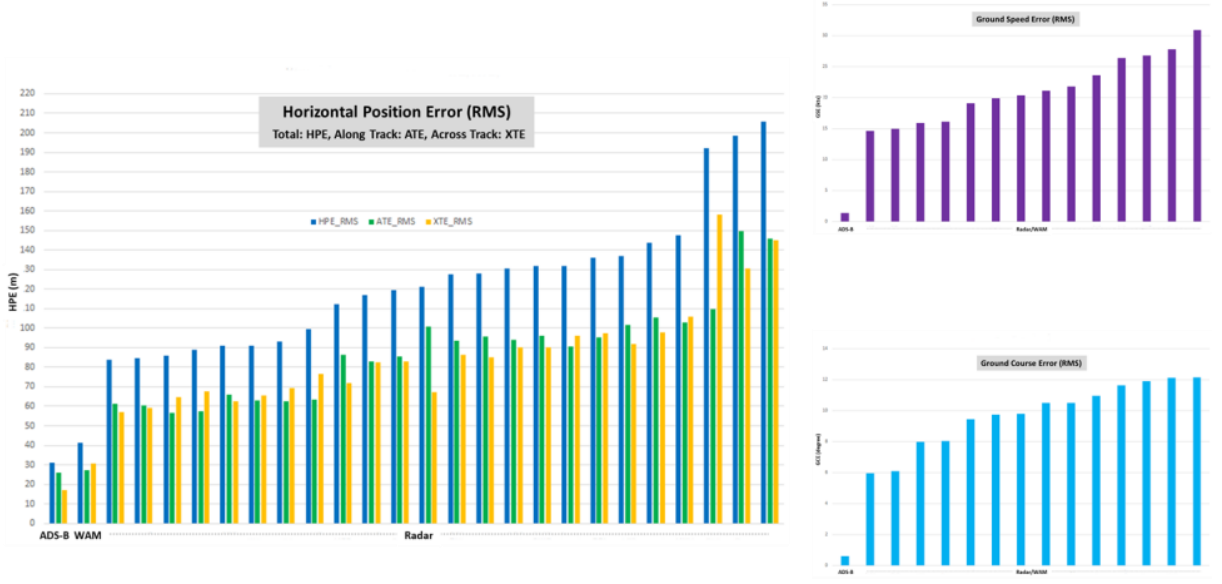

Figure 6 Sensor Performance Assessment

The comparative surveillance sensor performance assessment indicated that the horizontal position error and aircraft velocity/angle error is significantly improved by use of ADS-B data. Further performance parameter, which were analyzed: e.g. data transmission delay, track initiation delay, update period, probability of update and probability of sustained misses.

The SDPS in the DFS surveillance infrastructure use multi sensor/radar tracking systems (PHOENIX (DFS), ARTAS (EUROCONTROL)), which correlate and fuse the surveillance information from various sensors. The quality of surveillance data provided by conventional sensors (radar and multilateration) is already remarkable high. The existing surveillance systems providing redundant and seamless coverage. Therefore, the surveillance data processing system receive already high performance surveillance data, which allows a very precise tracking of targets. The introduction of an ADS-B sensor will further improve the tracking quality (see Figure 7).
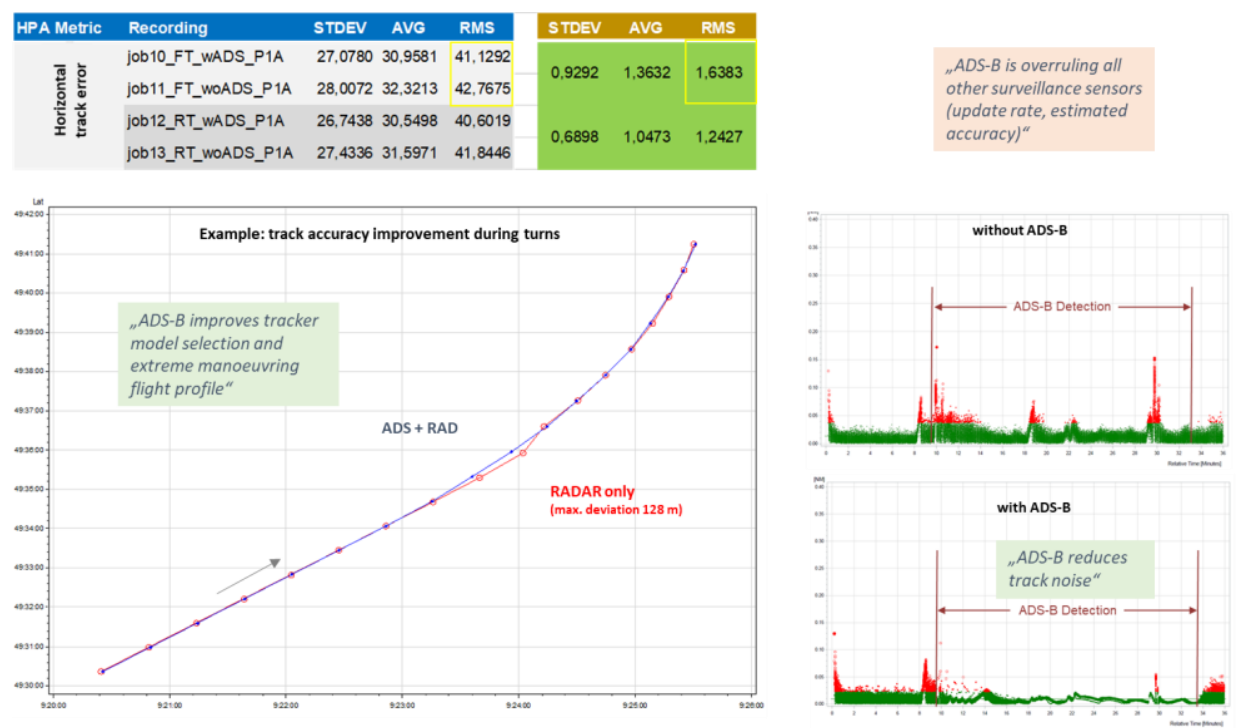

Figure 7 ADS-B Track Performance Assessment 


\section{ADS-B Safety Assessment (ASA)}

The ADS-B Safety Assessment conducted by DFS consisted of four Technical Workshops (technicians) and three Operational Workshops (ATCOs). The evaluated topics are follows: data quality, data rate, aircraft identification, security (e.g.: jamming, spoofing), identification for ADS-B unconfirmed Tracks and operational presentation of ADS-B information. The ADS-B security assessment was carried out during several national (LuFo: TeFiS) and international (SESAR 14.4.3) research projects (Dr. Heidelmeyer \& Dr. Hoffmann, 2008) (see Figure 8).

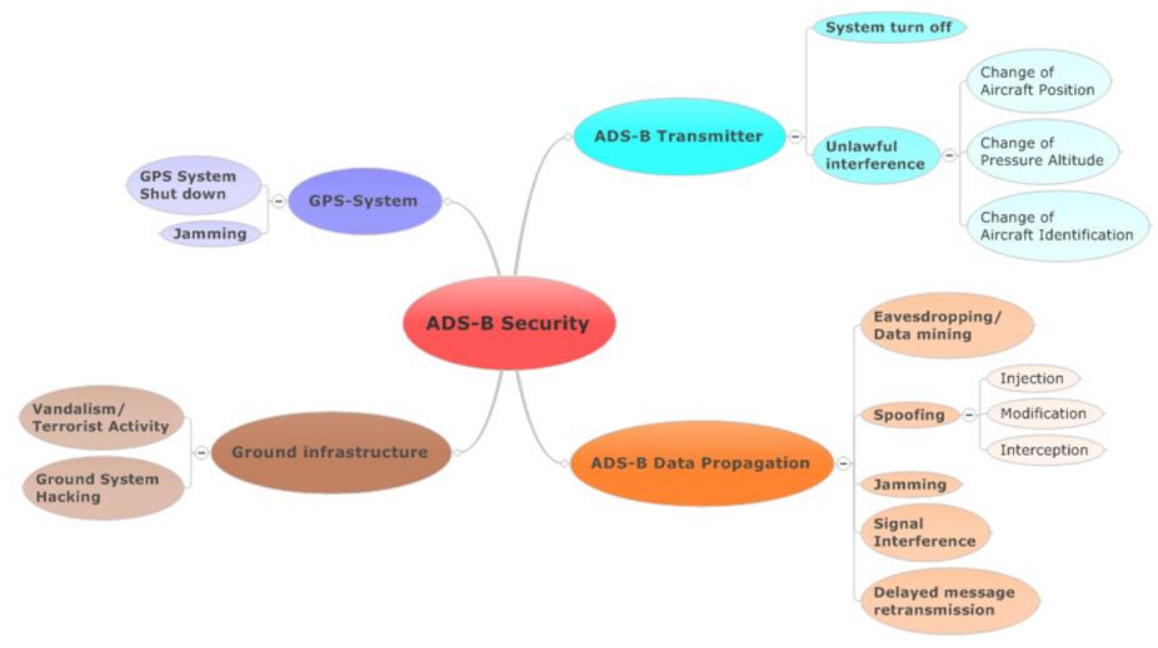

Figure 8 ADS-B Security Assessment

\section{ADS-B Validation Unit (AVU)}

With the surveillance technology ADS-B, the data is generated according to the principle of owndetection. This means that all data is determined by the respective object (aircraft, vehicle) itself, in particular its own position. The surveillance sensors used so far by air traffic control (radars, MLAT) work according to the principle of external external-detection according to which the position of the respective object is determined by a ground-based sensor. The quality of the position determination depends on the respective sensor. With external-detection, the performance parameters of the groundbased sensors are known and continuously monitored. With own-detection, the quality of the position determination depends on the respective on-board sensor of the target and is therefore no longer the responsibility of air traffic control. The ADS-B validation unit should guarantee the content integrity of the ADS-B data (positional data (horizontal position, barometric altitude) and non- positional data (ACID, flight status, etc.) with suitable methods.

The integrity of ADS-B data can be ensured either by comparison with data from other surveillance sensors (dependent validation) or by analyzing the properties of the data itself (independent validation), e.g. by means of simplified TDOA mechanisms. The independent validation of the ADS-B data appears to be a necessary prerequisite for providing a full Mode S Surveillance Layer based on ADS-B technology. In this context, the "ADS-B implementation concept at DFS" (V1.0, 2016) already provides for an ADS-B server in two possible forms (centralized, decentralized) for DFS's ADS-B architecture. Because of the examination of the architecture variants, a centralized "ADS-B Validation Unit" has now been identified as the most appropriate system design for the ADS-B server functionality. 
The ADS-B Validation Unit (AVU) consists of the actual Validation Module and further support modules (Input/Output, Reporting, Statistic and Control \& Monitoring). The Validation Module performs both dependent and independent validation of ADS-B data (see Figure 9).

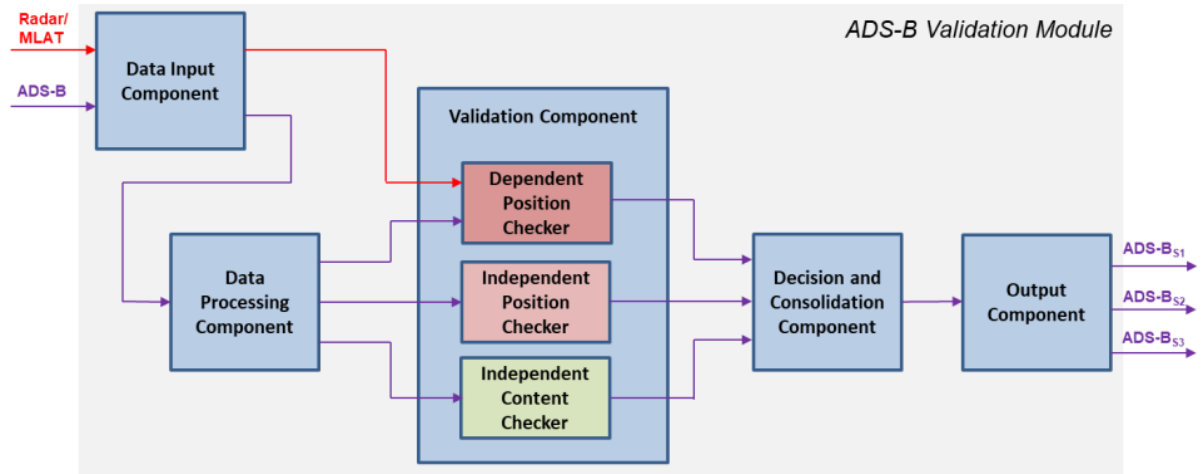

Figure 9 AVU functional overview

The module can be controlled by external Black/White (B/W) lists and has functions to generate $\mathrm{B} / \mathrm{W}$ lists automatically. The AVU also filters the data (geographical, quality parameters, ADS-B version, etc.) for the respective ADS-B services. The support modules of the AVU monitor their functions, provide information about the status of the ADS-B population (transponder deviation sheets may be generated) and generate the ADS-B data services.

\section{Experiences and Challenges}

Examples of the experiences gained during the ADS-B implementation are provided (list not exhaustive):

\subsection{Uncompensated Latency (mainly ADS-B MOPS version 0 und 1)}

In the ADS-B airborne system the GPS device receives signals from GPS satellites and determine the position of the aircraft including the time of applicability. The GPS position is relayed to the transponder either directly or via the flight management system. The uncompensated latency (UL) is

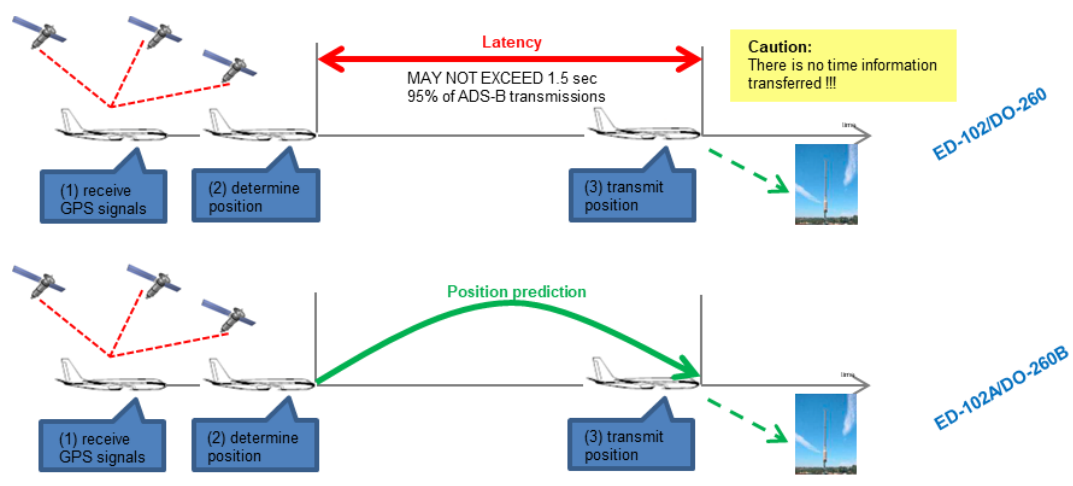

Figure 10 Along Track Deviation caused by UL 
the difference between the time of applicability for the transmitted position and the actual time the position is transmitted from the ADS-B airborne system.

The UL is depending various factors it various between different aircraft models and even in a dedicated aircraft is not a constant value. The problem of the UL occurs, because of the fact that the time of applicability of the position is not transferred with the ADS-B position messages (see Figure $10)$.

The UL of the ADS-B data has a significant influence on the Along Track Error (ATD) (see Figure 11). In the future, this deviation will be minimized by extrapolating the position to the transmission time or reduction of the transfer time (ADS-B version 2).
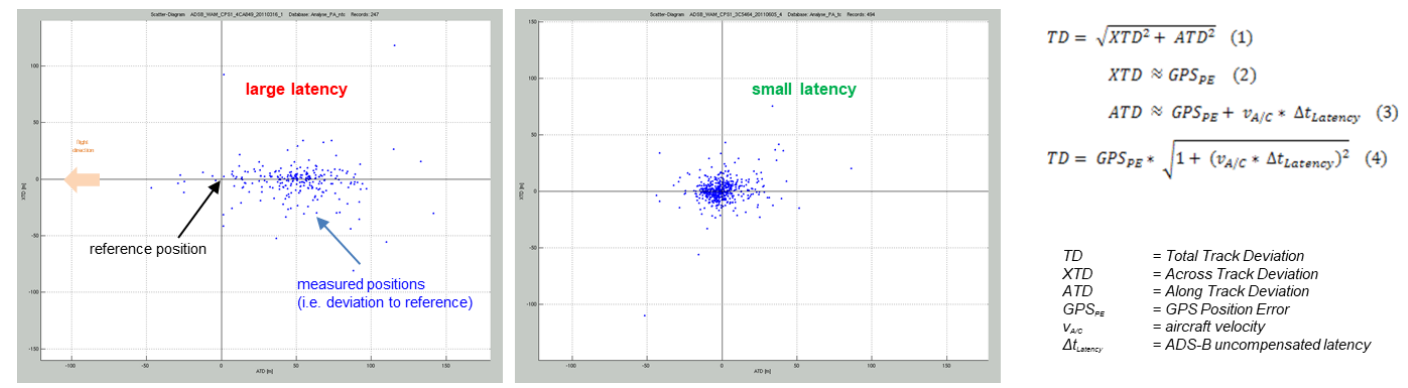

Figure 11 Uncompensated Latency

\subsection{Mode 3/A code "1000" (ADS-B MOPS version 2)}

The Mode 3/A code is transmitted every $5 \mathrm{sec}$ in the Aircraft Status Message (ASM) (see Figure 12). If a change of the Mode 3/A code occurs the update period of the ASM is reduced to one second

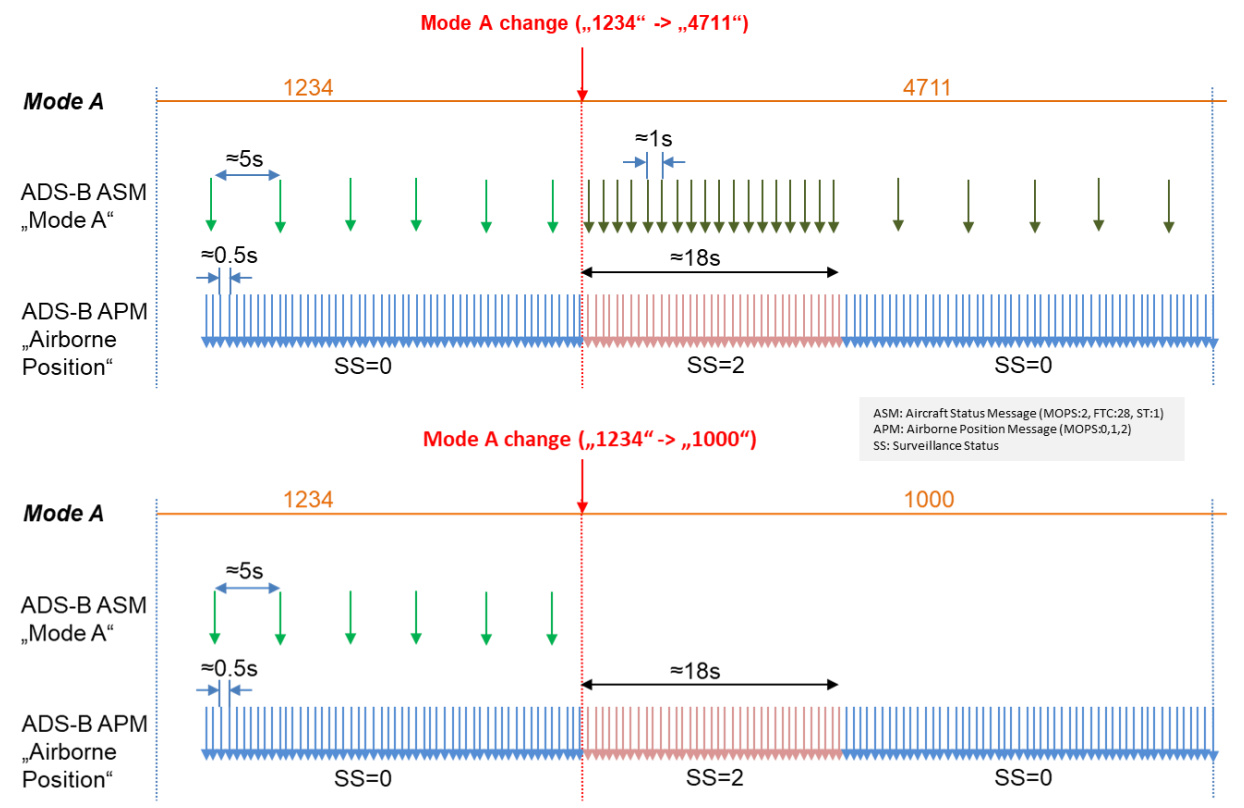

Figure 12 Change of Mode 3/A codes 
for a duration of $18 \mathrm{sec}$. In addition, the Surveillance Status (SS) flag is set to 2 (temporary alert). These allows the receiving systems to detect the Mode 3/A change quite fast.

In case of a Mode 3/A code of " 1000 " no ASM is transferred during ADS-B version 2 transmissions. The reason for this is to reduce the frequency load.

Because of the ADS-B version 2 is the mandated is the US and Europe, the missing ADS-B Mode 3/A " 1000 " code needs to be taken into account during the processing of the SDPS in order to prevent differing information. It has to be noted that the missing Mode 3/A code "1000" could not be derived from radar replies of the same target. Because only information contained in the Mode S ES messages are allowed to be used in the ADS-B reports created by the ground sensor. The missing Mode 3/A code may be supplemented in the SDPS.

\subsection{Transient Mode 3A codes}

If pilots do not deactivate the transmission of the new Mode 3/A code until the code change has been completed, Mode 3/A codes (transient), which have been set in the meantime will be transmitted (see Figure 13).

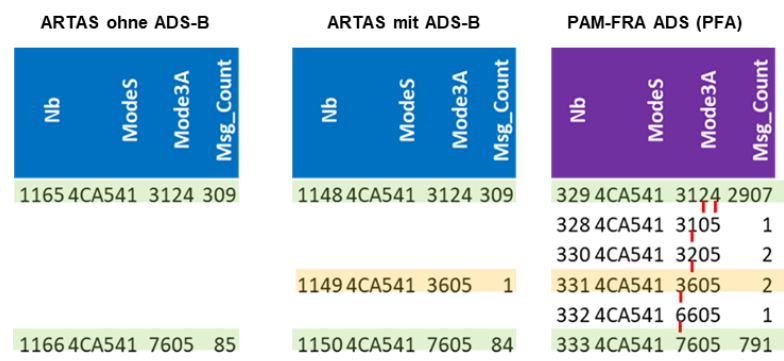

Figure 13 Transient Mode 3A codes (description)

The Mode 3/A code is used in SDPS as a mean of correlation between surveillance and flight plan data. If the Mode 3/A code is not correct (e.g. due to transient codes) a wrong or loss of correlation could be observed. Every fifth Mode 3/A change contain transient Mode 3/A codes. In the investigated area (see above PAM-FRA P1A), Mode 3/A changes occur rather rarely. It can be assumed that in other areas the absolute number of Mode 3/A changes can be significantly higher. It is also expected that at low altitudes the relative amount of transient Mode 3/A codes will increase. SDPS systems have to adjust to the occurrence of transient codes when processing data from sensors with higher update rates. In order to cope with the transient Mode 3/A a hysteresis of $2 \mathrm{sec}$ before a new Mode 3/A code is accepted is applied in SDPS.

\section{Conclusion}

The objectives are DFS ADS-B Implementation are as follows:

- Establishment of a third Mode S Surveillance Layer using ADS-B technology;

- The existing surveillance infrastructure (radar and multilateration) enables $3 \mathrm{NM}$ separation. The design goal is to use ADS-B as part of this multi sensor surveillance infrastructure;

- The existing safety and security level is at least to be maintained;

- The performance requirements (e.g. availability, accuracy and integrity) concerning the ADS-B system are at least as good as SSR (Mode-S radar) systems. 
DFS ADS-B Implementation in High Density Radar Controlled Airspace - Experiences ... S. Stanzel

The expected benefits of the DFS ADS-B Implementation are as follows:

- Medium-/long term economic benefits expected (e.g. less hardware and maintenance costs of the ADS-B sensors and - systems in comparison to radar systems taking into account the costs for data distribution and sites);

- Surveillance coverage in airspace possible (e.g. North Sea) hardly covered by radar;

- Potentially using higher update rates and better accuracy, e.g. in approach sectors with parallel runway approaches;

- Generation of surveillance information without (additional) radio field load, moreover reduction of radio field load possible (passive target acquisition) in case of potential substitution of SSR systems.

DFS has set up an ADS-B implementation program, which aims to coordinate all activities w.r.t. the introduction of the operational use of ADS-B in German airspace (Application of ADS-B RAD):

- Stepwise establishment of an ADS-B coverage (starting with upper airspace and local implementations);

- Modification of surveillance distribution, processing and display systems in order to process ADS-B data;

- Introduction of operational use of ADS-B data in DFS ATM systems (initially using only "confirmed" ADS-B targets);

- Implementation of a medium/long term cost efficient solution using multi sensor systems, which could be scaled to the optimum operational use;

- Multiple use of DFS sites; enable additional services bases on ADS-B surveillance data; potential use in airport environments.

\section{References}

Dr. Heidelmeyer, G., \& Dr. Hoffmann, H. (2008). Konzeption einer Sicherheitsbewertung zur Nutzung von ADS-B für eine Luftlagedarstellung zu Staffelungszwecken. Langen: DFS.

EASA. (2013). CS-ACNS (Certification Specifications and Acceptable Means of Complaince for Airborne Communications, Navigation and Surveillance) Initial Issue.

EUROCAE. (2009). ED-102A, Minimum Operational Performance Standards for $1090 \mathrm{MHz}$ Automatic Dependent Surveillance (Equivalent to RTCA DO-260B) (Equivalent to RTCA DO260B: Minimum Operational Performance Standards for $1090 \mathrm{MHz}$ Automatic Dependent Surveillance).

EUROCAE. (2009). ED-129B, Enhanced Air Traffic Services in Radar-Controlled Areas using ADS$B$ Surveillance (ADS-B RAD).

EUROCAE. (2009). ED-161, Enhanced Air Traffic Services in Radar-Controlled Areas using ADS-B Surveillance (ADS-B RAD).

EUROCONTROL. (2012). Eurocontrol Specification for ATM Surveillance System Performance (ESASSP), Volumes 1\&2, Edition Number 1.0, Document identity: Eurocontrol-Spec-0147 (and ISBN 978-2-87497-022-1).

EUROPEAN COMMISSION. (22. November 2011). Commission Implementing Regulation (EU) No 1207/2011 of 22 November 2011 laying down requirements for the performance and the interoperability of surveillance for the single European sky (with the dates amended through EU1028/2014). SPI/IR. Brussels: European Commission.

ICAO. (2012). Technical Provisions for Mode S Services and Extended Squitter (Doc 9871).

Stanzel, S. (April 2009). Konzept ADS-B Datenuntersuchung. Langen: DFS. 